\title{
Sexualidad y homosexualidad en la obra de Carlos Alberto Seguin.
}

\author{
Sexuality and homosexuality in the work of Carlos Alberto Seguin. \\ Jeff Huarcaya-Victoria ${ }^{1,2, a}$
}

\section{RESUMEN}

La sexualidad ha sido siempre un tema de interés tanto en círculos académicos como para el público en general. El concepto de "lo normal" en la sexualidad estuvo relacionado con cuestiones morales, religiosas o legales hasta el siglo XIX, a partir del cual se le comenzó a relacionar con conceptos y temas médicos. En comparación con otros psiquiatras de su época, Carlos Alberto Seguin dio mucha importancia al estudio de la sexualidad humana. Consideró que el desequilibrio de una personalidad puede verse reflejado directamente en su actividad psico-sexual, por lo cual es necesario indagar detenidamente acerca de élla. Las "desviaciones sexuales", con la homosexualidad como paradigma, suscitan tanto o más interés. La historia de la homosexualidad en el campo de la psiquiatría es compleja, algunos psiquiatras mantenían un enfoque de soporte y menos punitivo, mientras que otros jugaron un papel importante en el tratamiento médico de las conductas homosexuales. Seguin no consideró a la homosexualidad como una enfermedad, si bien la entendía como algo no deseable, contrapuesto al ideal de heterosexualidad. En base a su práctica clínica, postuló un sistema de clasificación de la homosexualidad y mantuvo una actitud comprensiva hacia ella, aun cuando no examinó de una manera directa las opiniones sociales y culturales sobre el tema, en el Perú de su época.

PALABRAS CLAVE: Sexualidad; homosexualidad; psiquiatría; Perú.

\section{SUMMARY}

Sexuality has always been a topic of interest both inside academic circles and among the general public. The concept of "normal" in sexuality was related to moral, religious or legal issues until the $19^{\text {th }}$. Century when it began to be relate to medical concepts and issues. Compared to other psychiatrists of his time, Carlos Alberto Seguin gave great importance to the study of human sexuality. He considered that an imbalanced personality can be directly reflected in his psycho-sexual activity, reason for which, it becomes necessary to carefully inquire about it. "Sexual deviations", with homosexuality as their paradigm arouse even more interest. The history of homosexuality in the field of psychiatry is complex, some psychiatrists maintained a supportive and less punitive approach, while others played an important role in the medical treatment of homosexual behaviors. Seguin did not consider homosexuality as an illness, although he understood it as something undesirable, opposed to the ideal of heterosexuality. On the basis of his clinical practice, he postulated a classification system of homosexuality, and maintained a sympathetic attitude toward it, even though he did not directly examine the social and cultural opinions about the topic during his life's work in Peru.

KEYWORDS: Sexuality; homosexuality; psychiatry; Peru.

1 Centro de Investigación en Salud Pública, Facultad de Medicina, Universidad de San Martín de Porres. Lima, Perú.

2 Departamento de Psiquiatría, Hospital Nacional Guillermo Almenara Irigoyen, EsSalud. Lima, Perú.

a Médico psiquiatra. 


\section{INTRODUCCIÓN}

Una de las figuras más representativas de la psiquiatría peruana fue Carlos Alberto Seguin (19071995), quien, desde el antiguo Hospital Obrero de Lima, actualmente Hospital Nacional Guillermo Almenara Irigoyen (HNGAI), reintrodujo las ideas psicodinámicas y psicosomáticas al cuerpo teórico psiquiátrico del Perú. La obra de Seguin se distingue por lo polifacético de su contenido. Para 1991 se documentó un total de 374 artículos de psiquiatría y disciplinas conexas, identificándose, entre otros tópicos, una tendencia a conceptualizar la medicina desde una perspectiva psicodinámica, diversas formas de psicoterapia, docencia médica y grupos formativos (1). Escribió además sobre sociología, antropología, arte y literatura. En un trabajo previo resaltamos la figura de Seguin, así como los aspectos fundamentales de su obra y legado (2). En este estudio revisaremos de algunas ideas que tuvo respecto a la sexualidad, en general, y la homosexualidad, en particular.

\section{El estudio de la sexualidad}

El espíritu poco misoneísta de Seguin le llevó a buscar el conocimiento para poder comprender y ayudar mejor a sus pacientes fuera de los límites de la medicina. Así, comenzó a frecuentar y entablar amistad con literatos, poetas, antropólogos, sacerdotes, sociólogos, filósofos, etc., a los cuales invitaba los sábados al HNGAI para realizar conferencias (3). Una de estas amistades se dio con el polímata Marco Aurelio Denegri Santagadea (1938-2018), a quien invitó al HNGAI en más de una oportunidad (Denegri publicó una de dichas conferencias, dictada en 1971, en la que disertó sobre la covada) (4). Ambos tenían un interés común, el estudio científico de la sexualidad, lo que les llevó a escribir sobre dicho tema no solo en el ámbito académico, sino también en forma de divulgación científica para el público no especializado. Seguin escribió las palabras liminares del libro de Denegri "Fascinum, ensayos sexológicos" (5) y colaboró además hasta en tres oportunidades en "Fascinum", la primera revista científica y artística de cultura sexual, dirigida por Denegri: En el número 1 con un trabajo "Sobre la clínica de la homosexualidad" (figura 1) (6), y en el número 9, con los artículos "El significado de la sexualidad humana" (7) y "La isla de las tres sirenas" (8).

La sexualidad constituye un aspecto central de todo ser humano, abarcando no solo el sexo, sino también las identidades y los papeles de género, el erotismo, el placer, la intimidad, la reproducción y la orientación

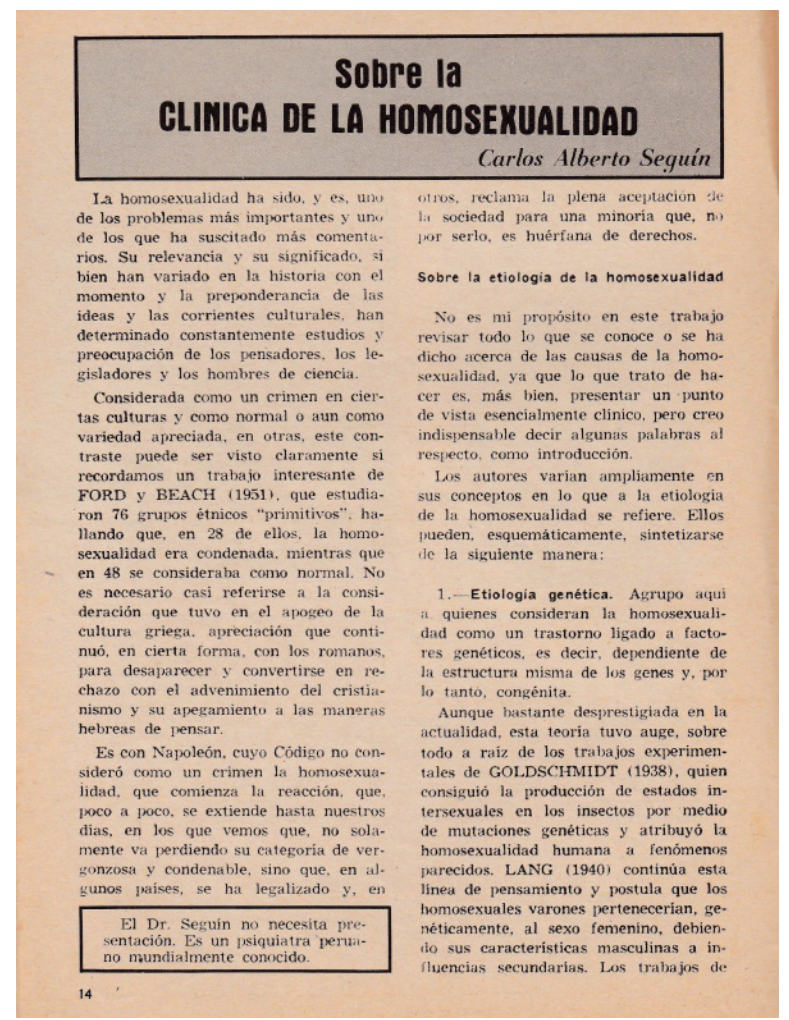

Figura 1. Artículo original "Sobre la clínica de la homosexualidad" (Biblioteca personal del autor). 
sexual, de tal manera que la sexualidad se encuentra influida por la interacción de factores biológicos, psicológicos, sociales, espirituales, económicos, políticos, culturales, éticos, legales, históricos y religiosos (9).

Respecto a lo sexual, Denegri mencionó que existe una contradicción histórica:

“... lo sexual y todo lo que con lo sexual se relaciona, despierta un extraordinario interés y tiene capital importancia en la vida de los hombres y de los pueblos; y, sin embargo -éste es el otro lado de la moneda- lo sexual ha sido objeto de un ocultamiento sistemático y constante, y tan manifiesto es el silencio cómplice en su torno como patente la inseguridad de ventilarlo pública y francamente." (10)

¿Cuáles son los factores que favorecen este "ocultamiento sistemático"?. Seguin escribió:

"Lo sexual no ha sido tabú siempre ni de la misma manera... Si la moral sexual en nuestros días debe sus características principalmente a la influencia de la Iglesia, y ésta a la reacción contra las costumbres de la Roma decadente, lo sexual se convierte en representación de lo material, de lo instintivo, de lo animal, que se enfrenta con lo espiritual.” (5)

Durante la primera mitad del siglo XX, el tema de la sexualidad en el Perú era tratado de una forma que actualmente consideraríamos como "puritana". Honorio Delgado consideraba que la sexualidad no tenía la importancia que se le atribuye, siendo las "incitaciones sexuales" parte de la "vida vulgar", todo lo cual debería ser superado para dar lugar a elementos más valiosos. Así, escribió en 1943:

“... con el sofrenar de las inclinaciones sensuales sin el cual no cobran energía las espirituales- organiza la vida interior y la dispone para la maduración de propiedades más positivas y valiosas. La disciplina y el vencimiento de sí, que entrañan renunciamiento a menudo doloroso, constituyen obra preliminar al dominio de sí mismo" (11).

Siguiendo un criterio teleológico o ideal de la normalidad en la materia sexual, Delgado citó a Schwarz:

"La única forma de actividad sexual normal es el coito fecundante dentro del matrimonio" (12).
Es interesante notar que en los trabajos de Delgado no se cite a uno de los pioneros del estudio científico de la sexualidad humana, Alfred Charles Kinsey (1894-1956). La primera edición del influyente "Curso de Psiquiatría", quizá una de las obras más trascendentales de Delgado, se publicó en 1953, fecha en la cual el trabajo de Kinsey ya era reconocido. Esta omisión podría tener hasta tres explicaciones: 1) Delgado tenía mayor consideración por los trabajos alemanes respecto a los estadounidenses; 2) los resultados de Kinsey no apoyaban la visión teleológica del estudioso peruano, y 3) causas políticas: las investigaciones de Kinsey sobre la sexualidad humana fueron blanco de duros ataques, no solo académicos ya que fue acusado de "ayudar al comunismo a minar la moralidad sexual y el carácter sacrosanto de los hogares norteamericanos" (actualmente existen grupos de personas que consideran a Kinsey un "degenerado", "pervertido", que trató de imponer la "nefasta ideología de género") (13).

Estas concepciones de la sexualidad comenzaron a cambiar durante la segunda mitad del siglo XX. Seguin tenía una opinión contraria a la de Delgado; para él, la sexualidad no se agota en la pura fecundación. Escribió en "Amor, sexo y matrimonio":

"Creemos que, en realidad, el sexo en la especie humana evolucionada se refiere a la unión corporal que, sin tener como fin único la reproducción, conlleva una serie de elementos afectivos de relación en los que al placer desinhibido se une el goce pleno de la entrega y la posesión" (14).

\section{Enfoques de Seguín}

Según Seguin debemos distinguir entre el ser y el significado de lo sexual. El ser está relacionado con la función sexual como una de las manifestaciones de la fisiología del individuo, siendo estudiada desde un punto de vista bioquímico, endocrinológico y neurológico (7). Mientras que el significado varía de acuerdo a distintos parámetros: 1) la cultura, existen culturas en las cuales lo sexual significa poco, mientras que, en otras, como la judeo-cristiana o la hindú, tiene gran significado, siendo considerado un aspecto de lo religioso; 2) los diferentes grupos socioeconómicos, citó a Kinsey, para quien el significado de la sexualidad varía de acuerdo al grupo socioeconómico en el cual nos encontremos, p.ej., para las clases elevadas la sexualidad se transforma en un problema moral, mientras que los de clase baja la racionalizan en base a lo que es natural o no natural, 
y 3) variaciones interindividuales, ya que el acto sexual puede tener significados distintos para cada persona y en cada momento (7). Seguin describió algunas variantes adicionales en cuanto al "uso" del sexo: 1) animal, el cual solo tenía fines reproductivos, siendo una actividad no humana, sino animal; 2) como deber u obligación dentro del matrimonio, y 3) para satisfacer necesidades neuróticas o para compensar la falta de otras satisfacciones.

Seguín enseñó cómo captar y evaluar una historia clínica de la vida sexual. Señaló que tomar los datos de la vida sexual es la parte más difícil del interrogatorio clínico, dificultad dada más que nada por la resistencia consciente o inconsciente que el médico presenta. El médico debe tener una actitud sana y comprensiva respecto a los problemas del sexo, lo cual se logra al tener la misma actitud frente a sus propios problemas sexuales. Sin embargo, en nuestra organización social, muchas veces el tabú del sexo dificulta que tanto hombres como mujeres puedan expresar sus problemas de forma comprensiva y seria. El médico, dice Seguin, para poder explorar adecuadamente la vida sexual de sus pacientes, debe tener una benevolente comprensión amistosa y segura, ya que, si hay inseguridad, falsa vergüenza, curiosidad malsana, "picardía", interés extra-medico, el paciente no podrá expresarse de forma natural y sincera (15).

Seguin escribió:

"Como se habrá notado, damos mucha importancia al interrogatorio sobre la vida sexual de nuestros pacientes. La experiencia nos ha enseñado que ello es indispensable. Piénsese lo que se quiera sobre las teorías psicoanalíticas, lo cierto es que el desequilibrio de una personalidad se refleja directamente en su actividad psico-sexual, la que puede servirnos de índice para el conocimiento de los problemas que esa personalidad enfrente y sobre su manera de resolverlos" (15).

\section{Homosexualidad y psiquiatría}

Se sabe que la sexualidad siempre ha sido un tema de interés tanto para el público lego como para los académicos. El concepto de "lo normal" en la sexualidad estuvo relacionado con cuestiones morales, religiosas o legales hasta el siglo XIX, desde el cual se comenzó a relacionar como un tema científico. No solo interesaron los patrones "normales" de la sexualidad, las "desviaciones" suscitan tanto o más interés. La homosexualidad es el paradigma de estas supuestas "desviaciones sexuales". Son muchos los factores que han sido relacionados con las actitudes hacia la homosexualidad; creencia en roles de género tradicionales, autoritarismo, dominancia social, religiosidad, etc., $(16,17)$.

La historia de la conceptualización de la homosexualidad en la psiquiatría resulta muy interesante, ya que nos muestra cómo los objetos de estudio de nuestra especialidad son híbridos (naturales y sociales), difiriendo así de las demás especialidades médicas (18). La homosexualidad comenzó a ser considerada como una enfermedad desde las publicaciones de Westphal (1870) y Krafft Ebbing (1886). Emil Kraepelin sostuvo hasta 1914-1915, una actitud notablemente progresista respecto a la homosexualidad y, junto con sus alumnos, contribuyó a la reformulación del Código Penal de su país y de la investigación psiquiátrica con la finalidad de proporcionar una mejor explicación de la sexualidad. La reorientación que experimentó luego de la primera guerra mundial y su posterior movimiento hacia la eugenesia hicieron que sus estudios psicosexuales no alcanzaran la maduración que alcanzaron sus estudios en psiquiatría clínica (19).

Durante la primera mitad del siglo XX, en el Jordanburn Nerve Hospital (JNH) de Edimburgo, existieron diversas estrategias de tratamiento para la homosexualidad: 1) en algunos casos se recurría a la hospitalización; 2) tratamiento de supresión hormonal; 3) cura de "reorientación". En aquel entonces existía un pobre consenso médico sobre la etiología y tratamiento de la homosexualidad, además, las actitudes de los psiquiatras hacia la homosexualidad permanecieron ambivalentes, algunos psiquiatras mantenían un acercamiento de soporte y menos punitivo, mientras que psiquiatras de otras instituciones, como la $\mathrm{JNH}$, jugaron un papel importante en el tratamiento médico de las conductas homosexuales (20).

En el Perú de aquella época, Delgado describió a la homosexualidad como:

“... la perversión que consiste en la búsqueda del orgasmo en el acceso carnal (paedicatio), en la masturbación mutua o en otra clase de actos lúbricos con individuos del mismo sexo, sin mayor interés erótico hacia el sexo opuesto y a menudo con la impresión de que el propio sexo fuera opuesto" (21).

Respecto a la génesis de la homosexualidad, Delgado mencionó que se debería a tres factores 
fundamentales: 1) los caracteres sexuales somáticos, es decir, el hermafroditismo físico; 2) los caracteres sexuales psíquicos, la actitud femenina en el varón y viceversa; y 3) la clase de elección del objeto sexual, o sea, optar por sujetos del propio sexo(12). El tratamiento era variado: régimen fisioterápico o gimnástico, hormonal, quirúrgico, cura psicoterapéutica desde la simple sugestión hasta el análisis proseguido por años (21). Aparte de los trabajos generales de Delgado sobre la vida instintiva, la sexualidad y sus desviaciones, llama la atención que, en la bibliografía psiquiátrica peruana, hayan sido pocos los médicos que estudiaron específicamente la homosexualidad (tabla 1) (22).

En este contexto, el trabajo de Seguin "Sobre la clínica de la homosexualidad" es un buen resumen del estado del arte que se tenía en su época sobre la homosexualidad. Seguin describe primero el contexto cultural de la homosexualidad, para luego resumir las principales teorías sobre su etiología. No considera a la homosexualidad como una enfermedad (en su artículo no figura la palabra "enfermedad"); ello no obstante, propone una clasificación y tratamiento de la misma.

Respecto a la clasificación de la homosexualidad, citó el trabajo de Kinsey, al que criticó por haber realizado solo una clasificación cuantitativa, más no cualitativa. El sentido pragmático de Seguin aflora en los siguientes párrafos, al proponer una clasificación sobre bases clínicas que el mismo elaboró luego de atender a varios pacientes, trabajo que presentó en un Congreso Internacional (Zurich, 1956):

1) Homosexuales latentes: Son aquellos pacientes que acuden al consultorio a buscar curación por síntomas que no tienen nada que ver con un posible trastorno sexual, como ansiedad o depresión, además de otros síntomas somáticos, como dispepsias, colitis, náuseas, vómitos, entre otros. Seguin escribió:

"La seria indagación psicológica descubre que se trata, en realidad, de homosexualidad reprimida. Los sueños, los actos fallidos, ciertas características del cuadro que el especialista avisado capta, le permiten descubrir el problema que no es otro que el de una homosexualidad que se mantiene latente y que produce la angustia ante el peligro, precisamente, de que se haga manifiesta." (6)

Esta irrupción de las tendencias homosexuales a la conciencia es lo que podría producir angustia. Respecto al tratamiento, postuló dos posibilidades; realizar una psicoterapia profunda, $y$, en caso de no poder realizarse ésta, se debería intentar un "tratamiento represivo", es decir, ayudar a ocultar estas tendencias (6).

2) Homosexuales en lucha: Son aquellos pacientes que comienzan a darse cuenta de cierta atracción homosexuales, lo cual les alarma, por lo que acuden al psiquiatra en busca de una "salvación" o "curación". Para Seguin, este grupo es el más dificultoso en cuanto al tratamiento, el cual consistiría en el esclarecimiento de los problemas reprimidos y la reestructuración de la enfermedad. En estos pacientes ya no es posible la represión, la cual ha sido obliterada por la fuerza de los impulsos homosexuales inconscientes (6).

3) Homosexuales decididos: Son aquellas personas que viven conscientemente su homosexualidad y no muestran angustia ante ella. Seguin escribió:

Tabla 1. Trabajos publicados en la bibliografía psiquiátrica peruana sobre la homosexualidad (22).

\begin{tabular}{|c|c|c|c|}
\hline Autor & Año & Nombre del artículo & Publicado en \\
\hline Gutiérrez-Noriega $\mathrm{C}$. & 1935 & $\begin{array}{l}\text { Historial y tratamiento de una } \\
\text { neurosis. Homosexualidad femenina } \\
\text { y complejo caracterológico ligado al } \\
\text { erotismo oral }\end{array}$ & $\begin{array}{l}\text { Anales de la Facultad de Ciencias } \\
\text { Médicas; } 17 \text { (2): } 277-392 \text { [Tesis para el } \\
\text { bachillerato en Medicina] }\end{array}$ \\
\hline Mayorga A. & 1956 & $\begin{array}{l}\text { Homosexualismo y esquizofrenia } \\
\text { paranoide }\end{array}$ & $\begin{array}{l}\text { Universidad Nacional Mayor de San } \\
\text { Marcos. } 176 \text { pp. [Tesis para el bachillerato } \\
\text { en Medicina] }\end{array}$ \\
\hline Saavedra V. & 1968 & $\begin{array}{l}\text { Homosexualismo secundario (a } \\
\text { propósito de un caso) }\end{array}$ & $\begin{array}{l}\text { Revista de la Sanidad Naval del Perú; 4(1): } \\
50-56\end{array}$ \\
\hline Seguin CA. & 1972 & Sobre la clínica de la homosexualidad & Fascinum; 1(1): 14-23 \\
\hline Rojo C. & 1974 & $\begin{array}{l}\text { Homosexualismo y esquizofrenia } \\
\text { paranoide }\end{array}$ & $\begin{array}{l}\text { Universidad Nacional Mayor de San } \\
\text { Marcos. } 122 \text { pp. [Tesis para el bachillerato } \\
\text { en Medicina] }\end{array}$ \\
\hline
\end{tabular}


"Estos casos no se presentan al médico por su desviación, que parece no ser problema para ellos, sino por otros síntomas: angustia y la cohorte de manifestaciones que he señalado en los pacientes del grupo 1: trastornos funcionales de diversos órganos y sistemas." (6)

En el grupo de homosexuales decididos, presentó el caso de "Pedro J.", mencionó que su insomnio estaba relacionado con la homosexualidad, pero no especificó como llego a dicha conclusión. Podemos suponer que, si bien Seguin no consideró a la homosexualidad como una enfermedad, sí la entendía como algo no deseable contrapuesto al ideal de heterosexualidad. Algo similar ocurrió con Freud, quien, si bien no consideró a la homosexualidad como entidad patológica, explicaba su origen en base al paradigma no cuestionado de la heterosexualidad como normalidad (23). Recordemos que al siguiente año de la publicación del artículo de Seguin "Sobre la clínica de la homosexualidad", la American Psychiatric Association retiró del "Manual diagnóstico y estadístico de los trastornos mentales" la etiqueta de homosexualidad.

¿Qué es entonces la homosexualidad para Seguin? No deja una idea clara al respecto en el mencionado artículo. Acaso, una forma de vivencia de la sexualidad humana que puede traer algún malestar en ciertas personas. Al hablarnos del tratamiento, se centró en el aspecto psicoterapéutico, no mediante "terapias de conversión" para que el paciente "regrese a la heterosexualidad", sino el esclarecimiento de las dudas básicas que tenga esta persona y, de ser posible, profundizar en el conflicto y aceptarlo. Destacó la actitud comprensiva que tuvo hacia este grupo sexual, si bien, no realizó una aproximación directa sobre las opiniones sociales y culturales peruanas respecto a la homosexualidad, las cuales son las generadoras de angustia en esta población. Es de resaltar la clara influencia psicoanalítica en la obra de Seguin, siendo esto evidente cuando habla de pulsiones, actos fallidos y el abrirse paso a la conciencia.

Finalmente, los diagnósticos en la psiquiatría rara vez desaparecen porque se presenten evidencias neurobiológicas en contra de ellos; esto sucede mas bien porque se vuelven socialmente peligrosos o incorrectos (24). Esto fue lo que pasó con el diagnóstico de la "homosexualidad", el cual luego de figurar por más de un siglo en las clasificaciones psiquiátricas desapareció de éstas al ser socialmente incorrecto considerar que una persona, por tener gustos sexuales "diferentes" deba ser considerada como enferma.
Recordemos que el concepto de enfermedad también tiene una dimensión política (25).

\section{CONCLUSIONES}

Carlos Alberto Seguin le dio mucha importancia al estudio de la sexualidad humana. Consideró que el desequilibrio de una personalidad puede verse reflejado directamente en su actividad psico-sexual, con lo cual, se hace necesario indagar sobre esta para conocer los problemas de esa personalidad enferma y su manera de resolverlos. Respecto a la homosexualidad, si bien no la consideró como una enfermedad, si la entendía como algo no deseable, contrapuesto al ideal de heterosexualidad. Postuló un sistema de clasificación de la homosexualidad en base a su práctica clínica. Mantuvo una actitud comprensiva hacia este grupo sexual, si bien, no realizó una aproximación directa sobre las opiniones sociales y culturales peruanas respecto a la homosexualidad.

\section{Correspondencia:}

Jeff David Huarcaya Victoria

Universidad de San Martín de Porres - Instituto de Investigación

Av. Alameda del Corregidor, 1531, La Molina, Lima Teléfono: (511) 3653640

Correo electrónico: jhuarcayav@usmp.pe_

Conflictos de interés: El autor declara no tenerlos

Agradecimientos: $\mathrm{El}$ autor desea expresar su más sincero agradecimiento al Dr. Santiago Stucchi Portocarrero por sus consejos y comentarios en la elaboración del presente artículo.

\section{REFERENCIAS BIBLIOGRÁFICAS}

1. Valdivia O. Panorama de la Psiquiatría en el Perú. Lima: Editorial Universidad Nacional Mayor de San Marcos; 1991.

2. Huarcaya-Victoria J. Carlos Alberto Seguin: a 75 años de su labor asistencial en el Hospital Nacional Guillermo Almenara Irigoyen. An Fac Med. 2016;77(4):403-8.

3. Silva M. Conversaciones con Seguin. Lima: Mosca Azul Editores; 1979.

4. Denegri M. Poliantea. Lima: Fondo Editorial de la Universidad Inca Garcilaso de la Vega; 2014.

5. Seguin CA. Palabras liminares. En: Denegri M, editor. Fascinum, Ensayos Sexológicos. Lima: Asociación de Estudios Humanísticos; 1972. p. 9-30.

6. Seguin CA. Sobre la clínica de la homosexualidad. 
FASCINUM. 1972;1(1):14-23.

7. Seguin CA. El significado de la sexualidad humana. FASCINUM. 1973;2(9):3-8

8. Seguin CA. La isla de las tres sirenas. FASCINUM. 1973;2(9):68-73.

9. Iacaponi R, Loyola F. Experiencia escolar de alumnos homosexuales de Liceos municipales de hombres. Tesis de licenciatura. Santiago: Universidad Academia de Humanismo Cristiano; 2013.

10. Denegri M. Fascinum, Ensayos Sexológicos. Lima: Asociación de Estudios Humanísticos; 1972.

11. Delgado H. La personalidad y el carácter. Lima: Editorial Lumen; 1943.

12. Delgado H. Psicología general y psicopatología de las tendencias instintivas. Rev Neuropsiquiatr. 1938;1(3):255-353.

13. León R. Literatura, Historia y Psicología en la obra de E.L. Doctorow PAIDEIA XXI. 2016;5(6):79-97.

14. Seguin CA. Amor, sexo y matrimonio. Lima: Ediciones ERMAR; 1980.

15. Seguin CA. Introducción a la Medicina Psicosomática. Lima: Empresa Gráfica T. Scheuch; 1947.

16. Whitley BE, Aegisdóttir S. The Gender Belief System, Authoritarianism, Social Dominance Orientation, and Heterosexuals' Attitudes Toward Lesbians and Gay Men. Sex Roles. 2000;42(11):947-67.
17. Huarcaya-Victoria J, Sancho Dávila-Palacios J, De la Cruz-Oré J. Relación entre la actitud hacia la homosexualidad y actitud religiosa en médicos de un hospital general. An Fac med. 2018;79(2):138-43.

18. Berrios GE. Psychiatry and its objects. Rev Psiquiatr Salud Ment. 2011;4(4):179-82.

19. Mildenberger F. Kraepelin and the 'urnings': male homosexuality in psychiatric discourse. Hist Psychiatry. 2007;18(71 Pt 3):321-35.

20. Davidson R. Psychiatry and homosexuality in midtwentieth-century Edinburgh: the view from Jordanburn Nerve Hospital. Hist Psychiatry. 2009;20(80 Pt 4):403-24.

21. Delgado H. Curso de Psiquiatría. Lima: Imprenta Santa Maria; 1953.

22. Valdivia O. Bibliografía Psiquiátrica Peruana. Lima: Talleres Litográfica; 1981.

23. Cornejo J. La homosexualidad como una construcción ideológica. Límite. 2007;2(16):83-108.

24. Berrios GE. Filosofía e historia de la Psiquiatría y la Psicopatología. Vertex (Argentina). 2011;22:5-18.

25. Reznek L. The Philosophical Defence of Psychiatry. Nueva York: Routledge; 1991.

Recibido: 09/10/2018

Aceptado: 09/12/2019 